\title{
Effect of Salinity on Hydroxyapatite Dissolution Studied by Atomic Force Microscopy
}

\author{
Ki-Young Kwon, Eddie Wang, Alice Chung, Neil Chang, and Seung-Wuk Lee* \\ Department of Bioengineering, University of California, Berkeley, Physical Biosciences Division, \\ Lawrence Berkeley National Laboratory, Berkeley Nanoscience and Nanoengineering Institute, \\ Berkeley, California 94720
}

Received: November 26, 2008; Revised Manuscript Received: January 29, 2009

\begin{abstract}
The complexity of bone tissue and the lack of techniques for directly probing bone surfaces in vivo have hindered studies on the fundamental mechanisms of bone mineral remodeling. Here, we addressed these issues by using single crystal hydroxyapatite (HAP) as a well-defined bone surface model and directly observe its surface using in situ atomic force microscopy. Specifically, we investigated the effects of $\mathrm{NaCl}$ concentration on the dissolution of HAP (100) surfaces and found that $\mathrm{NaCl}$ strongly suppressed HAP dissolution kinetics, including at physiological ion concentrations. These findings indicate that local ion concentrations in vivo may contribute significantly to the stability of bone mineral. Moreover, our analysis of HAP surfaces over a broad range of $\mathrm{pH}$ conditions suggests that only one kind of surface termination exists exclusively in the solutions we used. This observation is important for understanding the surface chemistry of HAP. Our molecular level, real-time observations of HAP dissolution are significant for understanding bone resorption and provide useful insights for the design of novel therapies for treating osteoporosis and other bone related diseases.
\end{abstract}

Bones are intricate, composite architectures composed of cells, extracellular macromolecules, and minerals. ${ }^{1}$ Continuous cell mediated remodeling of bone tissue by bone-resorbing osteoclasts and bone-depositing osteoblasts allows for the adaptation and maintenance of bone architecture, whereas imbalances result in critical bone diseases such as osteoporosis and Paget's disease. $^{2}$ Hence, a fundamental understanding of the bone remodeling process is needed to develop new therapies for treating such diseases. ${ }^{3}$ Besides the cellular aspects of the bone remodeling process, other important considerations are the surface chemistry of bone mineral and its interactions with the surrounding environment. Bone mineral is composed of biological apatite, a close analog of hydroxyapatite $\left(\mathrm{Ca}_{10}\left(\mathrm{PO}_{4}\right)_{6}(\mathrm{OH})_{2}\right)$ (HAP) ${ }^{4}$ The interactions of biological apatite with external ions and macromolecules dictate its morphology, and its growth and dissolution kinetics. ${ }^{5}$ However, the fundamental mechanism by which remodeling occurs on bone mineral surfaces remains elusive. Determination of these mechanisms has been hindered by the inherent complexity of bone tissue and the surrounding body fluid and by technological limitations associated with creating well-defined experimental systems.

As simplified models, dissolution and nucleation of many calcium phosphate crystals including synthetic and biological hydroxyapatite have been studied by measuring transient ion concentrations from bulk solutions. ${ }^{6}$ However, previous approaches could not address certain critical issues; namely, the dependence of dissolution/nucleation on surface structure. In addition, there is some ambiguity in the exact chemistry of the minerals being studied because much of the data based on bulk solution experiments were collected from ensembles of multiphase surfaces. Previously, to address this challenging problem,

* To whom correspondence should be addressed. E-mail: leesw@berkeley.edu. we investigated HAP dissolution processes by directly observing atomically well defined (100) HAP surfaces under acidic dissolution conditions using in situ atomic force microscopy $(\mathrm{AFM}) .{ }^{7} \mathrm{We}$ found that dissolution originating from small local structural defects (e.g., screw and edge dislocations) embedded on atomically flat terraces came to dominate the overall dissolution process by formation of hexagonal deep etch pits. Here, using the same technique, we report the effects of $\mathrm{NaCl}$ concentration on the HAP dissolution process. It should be noted that $\mathrm{Na}^{+} / \mathrm{Cl}^{-}$ions account for more than $90 \%$ of the ionic strength of human body fluid and thus these ions may significantly influence the dissolution of the ionic crystal of bone. However, the origin of these influences had not been determined. We found that $\mathrm{NaCl}$ has a strong ability to suppress HAP dissolution where, microscopically, both step retraction velocity and step density increase as $\mathrm{NaCl}$ concentration decreases. Dissolution proceeded by the formation of the hexagonal shapedpits with quantized height steps corresponding to the interlayer distance of the $(100)$ surface $(d(100) \approx 0.82 \mathrm{~nm})$ across a wide range of $\mathrm{pH}$ conditions.

Single crystal HAP was prepared through a molten salt synthesis as previously reported. ${ }^{8}$ The resulting crystals are elongated hexagonal rods mainly covered by six (100)-like surfaces (Figure 1, panels A and B). Surface morphology changes were imaged using a MFP3D AFM (Asylum Research, Santa Barbara, CA) in contact mode (spring constant of cantilever: $0.09 \mathrm{~N} / \mathrm{m}$ ) under different solution conditions. AFM images were first collected in pure water. Initially, the HAP (100) surface was dominated by atomically flat terraces separated by molecular steps (Figure 1C). By measuring the angles between the long axis of the crystal (100 direction of HAP) and the observed step lines, we assigned the orientation of individual steps on (100) surfaces. We found that steps parallel 


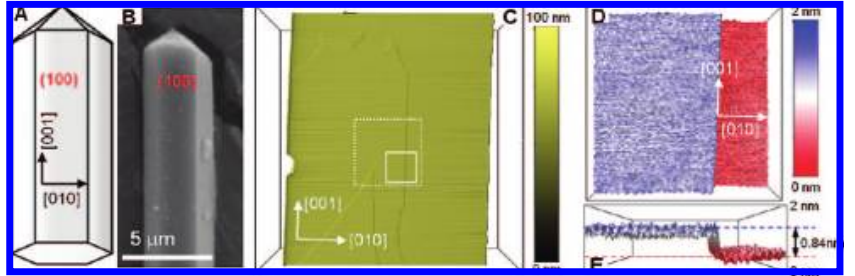

Figure 1. Surface structure of hydroxyapatite. (A) A model structure of an elongated hexagonal rod shaped HAP single crystal. The specific crystallographic directions and surfaces are indicated. (B) Scanning electron microscopy image of single crystal HAP. The HAP crystal is mainly covered by six equivalent (100)-like surfaces. (C) AFM image of single crystal (100) HAP surface (size: $7.0 \times 7.0 \mu \mathrm{m}$ ). The straight molecular step in the middle is parallel to the elongation direction of the crystal. The bar on the right represents the vertical scale. (D and E) Horizontal and vertical views of the two atomically flat terraces divided by the molecular step (solid boxed region in Figure 1C, size: $860 \times 860 \mathrm{~nm})$.

to the (001) direction were the most commonly observed in the analysis of more than fifty HAP samples. Panels D and E in Figure 1 show top down and side views of a step parallel to the [001] direction (boxed region in Figure 1C). Vertical crosssectional analysis shows that the average height of this step was $0.84 \pm 0.10 \mathrm{~nm}$, which corresponds to the interlayer distance between $(100)$ surfaces $(d(100) \approx 0.82 \mathrm{~nm})$. In pure water, no step retraction was observed due to dissolution for more than four hours before initiating dissolution using acid buffer solution (Supporting Information, Movie S1).

Subsequently, we investigated the evolution of the HAP (100) surface and the effect of $\mathrm{NaCl}$ on HAP dissoluton by replacing pure water with acidic buffer conditions ( $10 \mathrm{mM}$ citrate buffer, $\mathrm{pH}$ 6) containing $\mathrm{NaCl}$. We monitored the dashed boxed region in Figure $1 \mathrm{C}$ by constant AFM scanning for more than $70 \mathrm{~h}$ while sequentially flowing buffers having three different $\mathrm{NaCl}$ concentrations (1 M, $100 \mathrm{mM}$, and $10 \mathrm{mM}$ ) into the fluid cell. Specifically, using an electronically controlled syringe pump, at specified time points, the acidic buffers were rapidly switched from $1 \mathrm{M}$ to $100 \mathrm{mM} \mathrm{NaCl}$ and then later from $100 \mathrm{mM}$ to 10 $\mathrm{mM} \mathrm{NaCl}$ with essentially no stoppage in flow.

We observed multiple features on the HAP (100) surface during dissolution processes with three ionic concentration changes: First, throughout the entire dissolution process (Movie $\mathrm{S} 1$ ), dissolution proceeded by stochastic formation of elongated hexagonal-shaped flat-bottom etch pits with quantized step height corresponding to the interlayer distance of the (100) surface $(\mathrm{d}(100) \approx 0.82 \mathrm{~nm})$ and continued via the retraction of these steps (Figure 2A-C and Movie S1). Second, the hexagonalshaped pits are elongated in the (001) direction during the dissolution process, which is the same to the elongation direction of the HAP crystals. Third, the velocity of steps retracting parallel to the [001] direction is faster than steps parallel to the [010] direction. Therefore, pits merged more frequently along the [001] direction (Figure 2A) than along the [010] direction. Consequently, the step density across the [010] direction was greater than across the [001] direction (steps across the (100) and [010] directions are indicated by the red and blue tick marks, respectively, in Figure 2A). Fourth, as the ionic strength of $\mathrm{NaCl}$ was reduced, step velocity increased (Figure 2D). Finally, under lower $\mathrm{NaCl}$ concentration, step density also increases (Figure $2 \mathrm{E}$ ). Because generation of all steps is associated with new pit formation on flat terraces, higher step densities correspond to increased ease of dissolution from the terraces. Therefore, higher dissolution rates at lower $\mathrm{NaCl}$ concentration can be attributed to more favorable dissolution from both steps and terrace

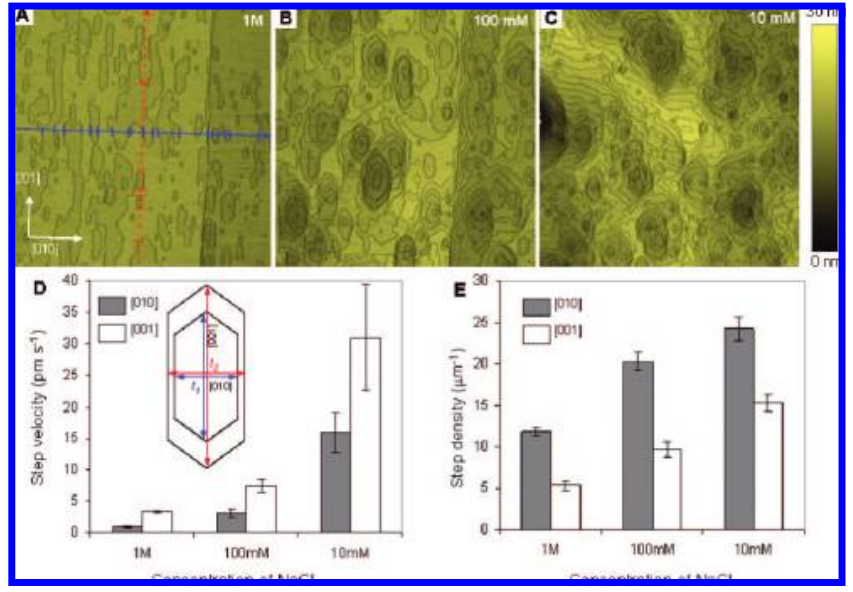

Figure 2. In situ characterization of single crystal (100) HAP surface affected by $\mathrm{NaCl}$ concentration at $\mathrm{pH}$ 6.0. (A-C) Three AFM images in $\mathrm{pH} 6$ buffer having $1 \mathrm{M}, 100 \mathrm{mM}$, and $10 \mathrm{mM} \mathrm{NaCl}$ concentration. (D) Dependence of step velocity on the $\mathrm{NaCl}$ concentration. Instead of measuring the retraction of steps from a fixed point as a reference, end to end distances (arrows in Figure 2D) of pits at different times ( $t$ ) are measured to exclude error due to thermal drifting. Step velocities are half of the pit growth velocity. Six to eight pits which occurred in temporally and spatially different conditions are analyzed at each $\mathrm{NaCl}$ concentration (error bars represent standard deviation). Two step velocities ( $\mathrm{pm} / \mathrm{s})$ along ([010], [001]) directions are $(0.95,3.3),(2.6$, $7.0)$, and $(16,30)$ at $1 \mathrm{M}, 100 \mathrm{mM}$, and $10 \mathrm{mM}$, respectively. (E) Dependence of step density on the $\mathrm{NaCl}$ concentration. Step density is determined by counting the number of steps per unit length across the [010] and [001] directions (blue and red tick marks in Figure 2a) in multiple regions. For each $\mathrm{NaCl}$ concentration, sixteen AFM images over a time interval of approximately $3 \mathrm{~h}$ are analyzed. Two step densities $\left(\mu \mathrm{m}^{-1}\right)$ along ([010], [001]) directions are $(12.2,5.75),(20.2$, $10.2)$, and $(25,16)$ at $1 \mathrm{M}, 100 \mathrm{mM}$, and $10 \mathrm{mM}$, respectively.

regions. We quantitatively measured the step velocities and step densities by analysis of sequential images in an AFM movie. The average dissolution rates that we calculated were $3.7( \pm 1.7)$ $\times 10^{-11}, 1.7( \pm 0.6) \times 10^{-10}$, and $1.1( \pm 0.6) \times 10^{-9} \mathrm{~mol} \mathrm{~m}^{-2}$ $\mathrm{s}^{-1}$ at $1 \mathrm{M}, 100 \mathrm{mM}$, and $10 \mathrm{mM} \mathrm{NaCl}$ concentrations respectively.

The occurrence of steps with quantized height on (100) HAP surfaces throughout the entire dissolution process suggests that only one kind of surface termination exists exclusively in the solutions we used. Determination of the HAP surface structure is critical to understanding the mechanisms of bone remodeling, which is not yet understood at the molecular level. Because of the complicated unit cell structure of hydroxyapatite crystals (ten $\mathrm{Ca}^{2+}$, two $\mathrm{OH}^{-}$, and six $\mathrm{PO}_{4}{ }^{3-}$ ), several different surface terminations have been postulated. ${ }^{9}$ Surface terminations with relatively high exposure of calcium ions or phosphate ions were proposed and considered positively and negatively charged surfaces, respectively (Figure 3A). In our comprehensive observation of (100) HAP surfaces, average step heights shorter than $d(100)$ were not observed during the entire dissolution process, which excludes the coexistence of the two layers in a given $\mathrm{pH}$ condition (Figure $\mathrm{S} 1$ ). In particular, at $1 \mathrm{M} \mathrm{NaCl}$ concentration, the dissolution rate is extremely slow (removal of 1 2 layers per day) and therefore we can rule out missing other step heights due to the time resolution limit of AFM scanning. Furthermore, when we switched the surface charge from positive to negative by gradually changing the $\mathrm{pH}$ across HAP's isoelectric point from 6 to 11.7 , we continuted to only observe $d(100)$ height steps (Figure 3B). The lack of observation of step heights shorter than $d(100)(1 / 3$ or $2 / 3$ 


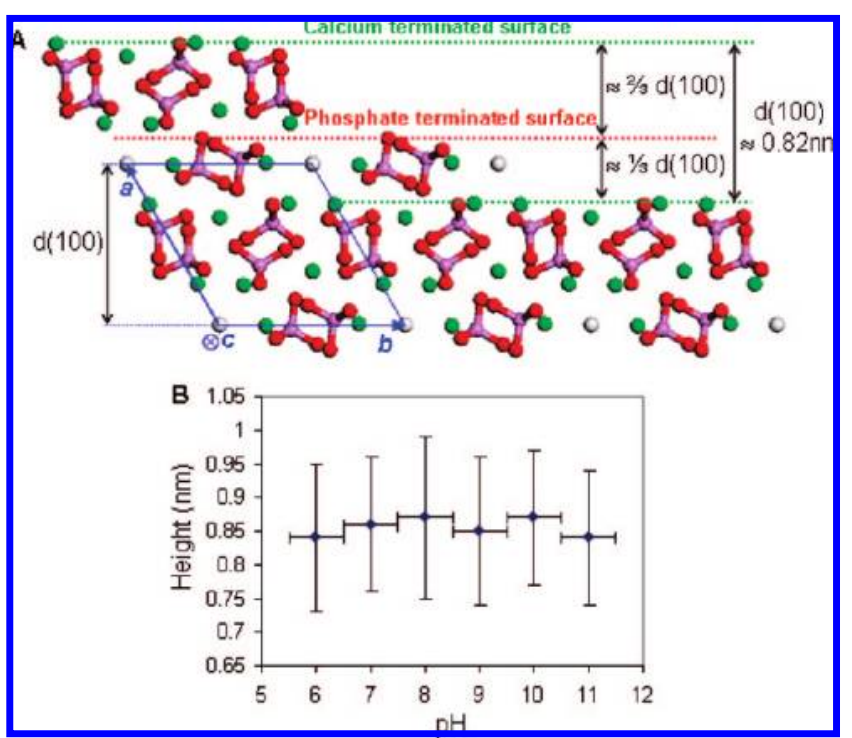

Figure 3. Surface termination of HAP. (A) HAP atomic models viewed along the [001] direction (Ca: green, O: red, P: purple, H: white). The conventional unit cell is represented by the blue diamond $(a=b=$ $0.9410 \mathrm{~nm}, c=0.6883 \mathrm{~nm}$ and $d(100) \approx 0.82 \mathrm{~nm})$. Among the (h00) surfaces, calcium and phosphate dominant surface terminations are indicated by dotted green and red lines, respectively. The absence of step heights less than $d(100)$ rules out the coexistence of calcium and phosphate dominant termination surfaces. (B) Step heights measured at different $\mathrm{pHs}$. $\mathrm{pH}$ is increased from 6 to 11.7 by injection of $\mathrm{pH}$ 11.7 solution slowly from the initial dissolution buffer condition $(\mathrm{pH}$ 6, $10 \mathrm{mM}$ citrate buffer). Average step heights range from 0.84 to 0.87 $\mathrm{nm}$, which correspond to $d(100)$.

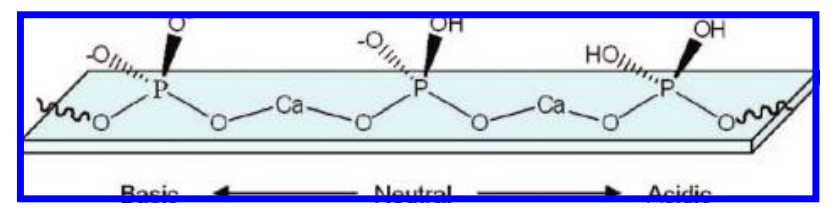

Figure 4. Schematic representation of surface protonation at different pHs.

of $d(100)$ in Figure $3 \mathrm{~A})$ under $\mathrm{pH} 6-11.7$ conditions implies that only one kind of surface termination exclusively exists in that $\mathrm{pH}$ range. This finding suggests that the HAP surface charge is more likely determined by the degree of protonation $^{10}$ of a specific apatite surface termination rather than by the interchange of a calcium or phosphate dominant surface (Figure 4). Previously, X-ray reflectivity was used to determine the surface termination of fluorapatite (FAP) in the water. $^{11}$ The proposed FAP/water interface is a Caand/or F-deficient outermost layer which is similar to the phosphate terminated surface in Figure 3A. If we hypothesize that FAP and HAP have same water interface, we propose that the phosphate terminated surface exists exclusively in the $\mathrm{pH}$ range from 6 to 11.7 .

Increases in ionic strength have been shown to either increase or decrease dissolution rates depending on the ionic crystal and ionic species. ${ }^{12}$ Therefore, there has been no general rule observed correlating the effect of ionic strength on mineral dissolution rates. Recent AFM based mechanistic studies demonstrated that steps play an important role in mineral dissolution and nucleation. ${ }^{13}$ Hence, investigations of step sources and of the factors affecting individual step velocities are important for understanding dissolution mechanisms and kinetics. Previously, we showed that HAP dissolution rate is dominated by local defects which become major sources of steps. ${ }^{7}$ In addition, many groups reported that dissolution rate can be significantly modified by changes of step velocities through interactions between additives (ranging from small inorganic ions to large peptides) and molecular steps. ${ }^{14}$ Particularly, selective interactions between additives and specific molecular steps caused the change of steps' orientations and even the bulk shapes of crystals. However, previously, we showed that pits with hexagonal shape formed on the (100) surface when dissolution experiments were performed in the same acidic buffer in the absence of $\mathrm{NaCl}^{7}$ In addition, hexagonal pit shapes were observed previously in both synthetic and biological apatites in various acidic solution environments without $\mathrm{NaCl} .{ }^{15}$ Our current experiment shows that the overall hexagonal pit shape is also retained in the presence of $\mathrm{NaCl}$, although well defined shapes are less obvious in the $10 \mathrm{mM} \mathrm{NaCl}$ condition due to multiple merges of individual steps and pits. The consistent observation of hexagonal pits in the presence of $\mathrm{NaCl}$ suggests that the mechanism by which $\mathrm{NaCl}$ inhibits HAP dissolution is not the result of strong interactions between $\mathrm{Na}^{+}$or $\mathrm{Cl}^{-}$ions and specific molecular steps. This is in accordance with previous bulk solution based studies which showed that unlike fluoride ions which are known to have a strong affinity to apatite surfaces, $\mathrm{Cl}^{-}$ions could not be incorporated into HAP through either ion exchange or adsorption in ambient aqueous solution. ${ }^{16}$ Furthermore, one obvious trend in HAP dissolution is that dissolution rate is increased as the solution $\mathrm{pH}$ decreases, which is a general phenomenon observed in dissolution of other minerals. This result indicates that dissolution proceeds by surface protonation which promotes breaking of calcium-oxygen bonds. Therefore, we propose that dissolution inhibition by $\mathrm{NaCl}$ is the result of competition for surface protonation sites between $\mathrm{Na}^{+}$and $\mathrm{H}^{+}$ions. This mode of inhibition caused by charge interaction occurs over the entire surface without preference for local surface structure. Our microscopic studies are consistent with this proposed mechanism because we observed inhibition of dissolution from both terraces and steps as evidenced by the decreases in step density and step velocity and because we did not observe any dependence on step direction as evidenced by the consistent hexagonal pit shape.

The effects of $\mathrm{NaCl}$ concentration on the surface dissolution process of HAP (100) surfaces were studied by real-time observation. Our findings suggest that typical $\mathrm{Na}^{+}$and $\mathrm{Cl}^{-}$ion concentrations $(140 \mathrm{mM})$ in extracellular body fluid may play a significant role in bone mineral stability. Based on our microscopic observations, we have proposed a mechanism for $\mathrm{NaCl}$ induced dissolution inhibition. Also, our observation of a single surface termination on HAP crystals shows that one kind of layer is exclusively exposed during the resorption process through a wide range of salt concentration and $\mathrm{pH}$ conditions. Therefore, the existence of one favorable surface should be considered on all HAP interactions with bone and tooth associated proteins. Further investigation of our well-defined HAP bone model surfaces with various amino acids and proteins will enhance our understanding of the molecular mechanisms of bone remodeling and provide useful insights for the design of novel therapies for osteoporosis, and many bone- and toothrelated diseases.

Supporting Information Available: Additional figures and a movie. This material is available free of charge via the Internet at http://pubs.acs.org. 


\section{References and Notes} 298

(1) Weiner, S.; Wagner, H. D. Annu. Rev. Mater. Sci. 1998, 28, 271-

(2) (a) Bronner, F.; Farach-Carson, M. C.; Rubin, J. Bone resorption: Springer: London, 2005. (b) Teitelbaum, S. L. Science 2000, 289, 15041508. (c) Rodan, G. A.; Martin, T. J. Science 2000, 289, 1508.

(3) (a) Kita, M.; Kondo, M.; Koyama, T.; Yamada, K.; Matsumoto, T.; Lee, K. H.; Woo, J. T.; Uemura, D. J. Am. Chem. Soc. 2004, 126, 4794 4795. (b) Vachal, P.; Hale, J. J.; Lu, Z.; Streckfuss, E. C.; Mills, S. G.; MacCoss, M.; Yin, D. H.; Algayer, K.; Manser, K.; Kesisoglou, F.; Ghosh, S.; Alani, L. L. J. Med. Chem. 2006, 49, 3060-3063. (c) Adamczyk, M.; Johnson, D. D.; Reddy, R. E. Angew. Chem. Int. Ed. 1999, 38, 35373539.

(4) (a) Elliott, J. C. Structure and chemistry of the apatites and other calcium orthophosphates; Elsevier: Amsterdam, The Netherlands, 1994. (b) Kay, M. I.; Young, R. A.; Posner, A. S. Nature 1964, 204, 1050.

(5) (a) Long, J. R.; Dindot, J. L.; Zebroski, H.; Kiihne, S.; Clark, R. H.; Campbell, A. A.; Stayton, P. S.; Drobny, G. P. Proc. Nat. Acad. Sci. 1998, 95, 12083-12087. (b) Song, J.; Malathong, V.; Bertozzi, C. R. J.Am. Chem. Soc. 2005, 127, 3366-3372. (c) Pan, H. H.; Tao, J. H.; Yu, X. W.; Fu, L.; Zhang, J. L.; Zeng, X. X.; Xu, G. H.; Tang, R. K. J.Phvs. Chem. B 2008, 112,7162 .

(6) (a) Tang, R. K.; Nancollas, G. H.; Orme, C. A. J. Am. Chem. Soc. 2001, 123, 5437-5443. (b) Christoffersen, J.; Christoffersen, M. R.; Kjaergaard, N. J. Crust. Growth 1978, 43, 501-511. (c) Jongeblo, W. 1.; Vandenbe, Pj; Arends, J. Calcif. Tissue Res. 1974, 15, 1-9.
(7) Kwon, K. Y.; Wang, E.; Chung, A.; Chang, N.; Saiz, E.; Choe, U. J.; Koobatian, M.; Lee, S. W. Langmuir 2008, 24, 11063-11066.

(8) Tas, A. C. J. Am. Ceram. Soc. 2001, 84, 295-300.

(9) (a) Astala, R.; Stottt, M. J. Phvs. Rev. B 2008, 78, 75427. (b) Sato, K.; Suetsugu, Y.; Tanaka, J.; Ina, S.; Monma, H. J. Colloid Interface Sci. 2000, 224, 23-27.

(10) Wu, L. M.; Forsling, W.; Schindler, P. W. J. Colloid Interface Sci. 1991, 147, 178-185.

(11) (a) Park, C. Y.; Fenter, P.; Zhang, Z.; Cheng, L. W.; Sturchio, N. C. Am. Mineral. 2004, 89, 1647-1654. (b) Pareek, A.; Torrelles, X.; Rius, J.; Magdans, U.; Gies, H. Phvs. Rev. B 2007, 75, 35418.

(12) Sahai, N.; Sverjensky, D. A. Geochim. Cosmochim. Acta 1997, 61, $2827-2848$.

(13) (a) Teng, H. H.; Dove, P. M.; Orme, C. A.; De Yoreo, J. J. Science 1998, 282, 724-727. (b) Dove, P. M.; Han, N. Z.; De Yoreo, J. J. Proc. Nat. Acad. Sci. 2005, 102, 15357-15362.

(14) (a) Fu, G.; Qiu, S. R.; Orme, C. A.; Morse, D. E.; De Yoreo, J. J. Adv. Mater. 2005, 17, 2678. (b) De Yoreo, J. J.; Dove, P. M. Science 2004, 306, 1301-1302.

(15) (a) Dorozhkin, S. V. J. Crust. Growth 1997, 182, 125-132. (b) Daculsi, G.; Legeros, R. Z.; Mitre, D. Calcif. Tissue Int. 1989, 45, 95-103. (c) Voegel, J. C.; Frank, R. M. Calcif. Tissue Res. 1977, 24, $19-27$.

(16) (a) Sugiyama, S.; Fukuda, N.; Matsumoto, H.; Hayashi, H. N.; Shigemoto, N.; Hiraga, Y.; Moffat, J. B. J. Colloid Interface Sci. 1999, 220, 324-328. (b) Krajewski, A.; Ravaglioli, A.; Roveri, N.; Bigi, A.; Foresti, E. J. Mater. Sci. 1990, 25, 3203-3207.

JP810414Z 